\title{
327.
}

\section{ON THE STEREOGRAPHIC PROJECTION OF THE SPHERICAL CONIC.}

[From the Philosophical Magazine, vol. xxv. (1863), pp. 350-3533.]

IN order to the tolerable delineation of some figures relating to spherical geometry, I had occasion to consider the stereographic projection of the spherical conic. To fix the ideas, imagine a sphere having its centre in the plane of the paper, and through the centre three rectangular axes, that of $x$ horizontal and that of $y$ vertical, in the plane of the paper, and the axis of $z$ perpendicular to and in front of the plane of the paper. The radius of the sphere is taken equal to unity (so that its intersection by the plane of the paper is the circle radius unity), and the points $X, Y$, and $Z$ are taken to denote the points where the axes, drawn in the positive direction, meet the surface of the sphere; and the opposite points are called $X^{\prime}, Y^{\prime}$, and $Z^{\prime}$. The eye is supposed to be at $Z$, and the projection to be made on the plane of the paper. This being so, and supposing that the axes of coordinates are the principal axes of the spherical conic, the axis of $x$ being the interior axis, and taking $\xi, \eta, \zeta$ as the coordinates of a point on the spherical conic, its equations are

$$
\begin{gathered}
\xi^{2}+\eta^{2} \quad+\zeta^{2}=1, \\
-\xi^{2}+\eta^{2} \cot ^{2} \beta+\frac{\zeta^{2}}{c^{2}}=0 ;
\end{gathered}
$$

where it may be remarked that $\tan \beta, c$ are the semiaxes of the plane conic which is the gnomonic projection (i.e. the projection by lines through the centre of the sphere) of the spherical conic on the tangent plane at $X$ or $X^{\prime}$.

Taking, for a moment, $x, y, z$ as the coordinates of a point on the projecting line (that is, the line through the eye to a point $(\xi, \eta, \zeta)$ on the spherical conic), the equation of this line is

$$
\bar{\xi}=\frac{y}{\eta}=\frac{z-1}{\zeta-1}
$$


and thence putting $z=0, x, y$ will be the coordinates of a point of the projection, and we hâve

$$
\frac{x}{\xi}=\frac{y}{\eta}=\frac{1}{1-\zeta}
$$

or, what is the same thing,

$$
\xi=x(1-\zeta), \quad \eta=y(1-\zeta)
$$

the equations of the spherical conic may be written

$$
\begin{aligned}
1-\zeta^{2} & =\xi^{2}+\eta^{2}, \\
\zeta^{2} & =c^{2}\left(\xi^{2}-\eta^{2} \cot ^{2} \beta\right) ;
\end{aligned}
$$

and by eliminating $\xi, \eta, \zeta$ fron the four equations, we obtain the equation of the conic.

Substituting for $\xi$ and $\eta$ their values, we find

$$
\begin{aligned}
1+\zeta & =\left(x^{2}+y^{2}\right)(1-\zeta), \\
\zeta^{2} & =c^{2}\left(x^{2}-y^{2} \cot ^{2} \beta\right)(1-\zeta)^{2} ;
\end{aligned}
$$

or, observing that the first equation gives

$$
\zeta=\frac{x^{2}+y^{2}-1}{x^{2}+y^{2}+1}
$$

and that thence

$$
1-\zeta=\frac{2}{x^{2}+y^{2}+1}, \quad \frac{\zeta}{1-\zeta}=\frac{1}{2}\left(x^{2}+y^{2}-1\right)
$$

the equation is

$$
\left(x^{2}+y^{2}-1\right)^{2}=4 c^{2}\left(x^{2}-y^{\grave{2}} \cot ^{2} \beta\right) .
$$

It is now very easy to trace the curve. We see first that the curve is symmetrical with respect to the axes, and that it meets the axis of $y$ in four imaginary points, but the axis of $x$ in four real points, the coordinates whereof are

$$
x= \pm\left(\sqrt{1+c^{2}} \pm c\right),
$$

so that the two points on the same side of the centre are the images one of the other in regard to the circle radius unity. Moreover the curve touches the lines

$$
y= \pm x \tan \beta
$$

at their intersections with the circle. By developing in regard to $y$, the equation becomes

and putting

$$
y^{4}+2\left(x^{2}-1+2 c^{2} \cot ^{2} \beta\right) y^{2}+\left(x^{2}-1\right)^{2}-4 c^{2} x^{2}=0 ;
$$

$$
x= \pm\left(\sqrt{1+c^{2}} \pm c\right),
$$


the last term vanishes, and the equation gives $y^{2}=0$, or

$$
\begin{aligned}
y^{2} & =2\left(1-x^{2}-2 c^{2} \cot ^{2} \beta\right) \\
& =4\left(-c^{2} \mp c \sqrt{1+c^{2}}-c^{2} \cot ^{2} \beta\right) \\
& =4 c\left(-c \operatorname{cosec}^{2} \beta \mp \sqrt{1+c^{2}}\right),
\end{aligned}
$$

the upper sign corresponding to the exterior values

$$
\pm x=\sqrt{1+c^{2}}+c
$$

and the lower sign to the interior values

$$
\pm x=\sqrt{1+c^{2}}-c .
$$

In the former case the values of $y$ are imaginary; in the latter case they are real if

$$
\sqrt{1+c^{2}}>c \operatorname{cosec}^{2} \beta \text {, }
$$

or, what is the same thing, if

$$
\sin ^{2} \beta>\frac{c}{\sqrt{1+c^{2}}}
$$

that is, if (for a given value of $c$ ) $\beta$ is sufficiently great, but otherwise they are imaginary.

If, as in the annexed figures, $c=\frac{5}{12}$ (and therefore $\sqrt{1+c^{2}}=\frac{13}{12}, \sqrt{1+c^{2}}+c=\frac{3}{2}$,

Fig. 1.

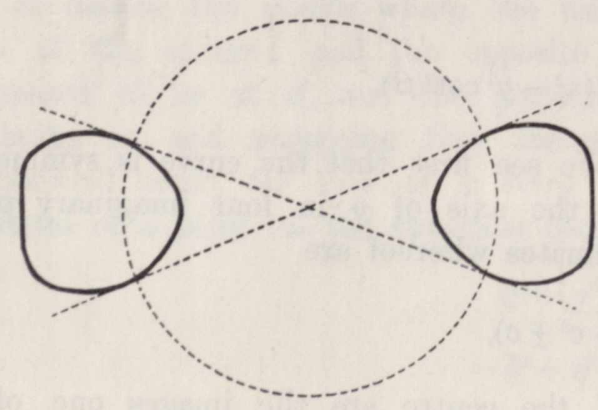

Fig. 2.

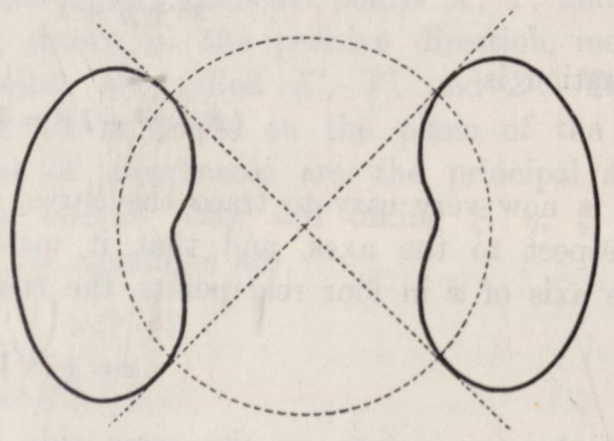

$\sqrt{1+c^{2}}-c=\frac{2}{3}$ ), then for the limiting value of $\beta$ we have

$$
\sin ^{2} \beta=\frac{5}{13}=\cdot 3846, \sin \beta=\cdot 62 \text {, or } \beta=38^{\circ} \text { nearly. }
$$

In the first figure $\beta$ is less, in the second figure greater than this value: the form for the limiting value is obvious from a comparison of the two figures.

I take the opportunity to mention the following theorem, which is perhaps known, but I have not met with it anywhere; viz. any three circles, each two of which meet, 
may be considered as the stereographic projections of three great circles of the sphere. In fact suppose, as above, that the projection is made on the plane of a great circle, and calling this the principal circle, the projection of any other great circle meets the principal circle at the extremities of a diameter of the principal circle. It follows that the theorem will be true, if, given any three circles each two of which meet, a circle can be drawn meeting the given circles, each of them at the extremities of a diameter of the circle so to be drawn. It is easy to see that the required circle has for its centre the radical centre (point of intersection of the radical axes) of the given circles, and that the radius is the 'Inner Potency' of the point in question in regard to each of the three given circles. In particular the three circles having for centres the vertices of an equilateral triangle, and the side for radius, may be considered as the stereographic projections of three great circles of a sphere. This is a very ready mode of delineation of a spherical figure depending on three great circles of the sphere.

2, Stone Buildings, W.C., March 21, 1863. 